\title{
Rol del tratamiento sistémico en el cáncer gástrico
}

\author{
Role of systemic treatment in gastric cancer
}

\author{
Natalia Ferrari, M.D. ${ }^{\prime}$
}

\begin{abstract}
Resumen
El cáncer gástrico en estadios tempranos es curable con cirugía, pero en muchos otros casos es mortal. La adición de quimioterapia y radioterapia a la cirugía ha mejorado la supervivencia general en estadios localizados. Los tratamientos sistémicos, que incluyen quimioterapia, inmunoterapia, anticuerpos monoclonales, otras terapias dirigidas, o combinaciones de éstas se están usando en estadios avanzados para mejorar la supervivencia y paliar síntomas.
\end{abstract}

Palabras clave: Cáncer gástrico, tratamiento adyuvante, tratamiento neoadyuvante, tratamiento de metástasis, tratamiento sistémico.

Abstract

Gastric cancer can be cured surgically when found in early stages, but it is still a deadly disease in many cases, especially when found in more advanced stages. Systemic and regional treatments have been added to surgery to improve the outcomes in localized disease. Systemic treatments are also used in advanced or metastatic disease to improve survival y palliate symptoms. Cytotoxic therapy, immunotherapy, monoclonal antibodies, other targeted therapies, and combinations of these have improved the survival in the metastatic setting.

Keywords: Gastric cancer, adjuvant treatment, neoadjuvant treatment, treatment of metastatic disease, systemic treatment.

\section{Introducción}

El cáncer gástrico puede ser curado quirúrgicamente en estadios tempranos, pero sigue siendo una enfermedad altamente letal. La tasa de supervivencia a 5 años en EEUU del 2010 al 2016 para un estadio localizado fue de $69 \%$ y cayó a menos del $35 \%$ para los estadios II en adelante ${ }^{(1)}$.

La tasa de mortalidad del cáncer de estómago en el Perú es de 19.3 por 100000 habitantes $^{(2)}$.

El estadiaje del cáncer gástrico se hace usando el sistema TNM del American Joint Commision on Cancer $(\mathrm{AJCC})^{(3)}$. Los estadios I a III, basados en la invasión de la pared gástrica y compromiso de ganglios linfáticos regionales, son estadios tempranos, potencialmente curables. El estado IV denota enfermedad metastásica, para la cual no hay tratamiento curativo hasta la fecha.
Los tratamientos sistémicos (como quimioterapia e inmunoterapia) y regionales (radioterapia) se han añadido al tratamiento quirúrgico con la finalidad de aumentar la supervivencia. El cáncer gástrico avanzado (cuando la enfermedad no es resecable o tiene metástasis) se trata por vía sistémica.

\section{Rol de los tratamientos sistémicos y regionales en el cáncer gástrico temprano}

La tasa de recurrencia local y distante después de la cirugía con intención curativa ha sido de 40 y $60 \%$ respectivamente ${ }^{(4)}$. Para disminuir la recurrencia se ha añadido tratamientos sistémicos y regionales, dados después de la cirugía (adyuvantes), antes de la cirugía (neoadyuvantes) o peri-operatoriamente.

${ }^{1}$ Médico-Cirujana, Hematóloga, Oncóloga. ORCID ID: 0000-0002-5641-7273 


\section{Quimio-Radioterapia (QRT) adyuvante}

En 1993 el metanálisis dirigido por J. Hermans que incluyó 2096 pacientes, concluyó que la QRT adyuvante no mejoraba la supervivencia en pacientes tratados quirúrgicamente $^{(5)}$, pero en 1998 Zhang, et al. presentaron un estudio randomizado comparando radioterapia neoadyuvante con cirugía sola $^{(6)}$. El estudio demostró diferencias significativas en supervivencia, tasa de resecabilidad, tasa de metástasis a ganglios regionales y tasa de recurrencia, favoreciendo al grupo tratado con radioterapia neoadyuvante.

Este resultado positivo usando radioterapia, llevó a considerar la combinación de radioterapia con quimioterapia dados concurrentemente (quimioradioterapia QRT). Así, en el 2001, J. McDonald, et al. publicaron los resultados del estudio INT-0116, comparando QRT adyuvante con cirugía sola ${ }^{(7)}$. La QRT usó 5-fluorouracilo (5FU) y leucovorina (LV). La supervivencia media fue de 36 meses para el grupo de QRT y 27 meses para el grupo de cirugía sola. La tasa de supervivencia a 3 años fue de $50 \%$ con QRT y $41 \%$ con cirugía sola. El HR (Hazard ratio/cociente de riesgo) de morir fue 1.35 (CI/intervalo de confianza 95\%, 1.09-1.66; $\mathrm{P}=0.005)$ y el $\mathrm{HR}$ de recurrencia fue 1.52 (CI 95\%, 1.23-1.86; $\mathrm{P}<0.001$ ), favoreciendo al grupo de QRT. La supervivencia libre enfermedad fue de 30 meses para el grupo de QRT y 19 meses el grupo de cirugía sola. La supervivencia sin enfermedad a 3 años fue de $48 \%$ en el grupo de QRT y $31 \%$ en el grupo con cirugía sola. La recurrencia del cáncer fue reportada en $64 \%$ de los pacientes con cirugía sola y $43 \%$ en los del grupo de QRT.

El estudio del Cáncer and Leukemia group (CALGB 80101) comparó el tratamiento del estudio INT-0116 (QRT con $5 \mathrm{FU})$ con $\mathrm{QRT}$ con un régimen más intenso usando epirubicina (E), cisplatino $(\mathrm{C})$ y $5 \mathrm{FU}(\mathrm{ECF})^{(8)}$. Este estudio demostró una supervivencia general y supervivencia sin enfermedad similar a INT-0116.

El estudio ARTIST comparó quimioterapia adyuvante (capecitabina y cisplatino) con QRT adyuvante en pacientes que habían tenido resección completa y linfadenectomía D2 ${ }^{(9)}$. (Linfadenectomía D1 incluye los ganglios perigástricos, mientras que la D2 se extiende, además, hacia los grupos ganglionares de la arteria gástrica izquierda, arteria hepática común, tronco celíaco, hilio esplénico y arteria esplénica). Aquí, la adición de radioterapia no mejoró la supervivencia sin enfermedad o supervivencia general comparada con quimioterapia adyuvante sola en pacientes que habían tenido resección gástrica y linfadenectomía D2. El estudio ARTIST-2 tampoco demostró beneficio de la QRT comparada con quimioterapia adyuvante en el grupo de pacientes con ganglios linfáticos positivos que fueron tratados con linfadenectomía $\mathrm{D} 2^{(10)}$.

\section{Quimioterapia adyuvante}

La quimioterapia adyuvante también ha demostrado beneficio en comparación con cirugía sola. El estudio ACTSGC usando S1 adyuvante (una fluoropirimidina oral) demostró un aumento de la supervivencia general en comparación a cirugía sola ${ }^{(11)}$ y el estudio CLASSIC, usando capecitabina y oxaliplatino adyuvante, mejoró la supervivencia libre de enfermedad a 3 años y supervivencia general a los 5 años ${ }^{(12)}$.

\section{Quimioterapia perioperatoria}

El estudio MAGIC utilizó la combinación de epirubicina, cisplatino y 5FU (ECF) perioperatoriamente y comparó quimioterapia perioperatoria con cirugía sola ${ }^{(13)}$. El grupo de quimioterapia tuvo más resecciones con márgenes libres (R0). El 90\% de los pacientes asignados a quimioterapia completaron el tratamiento preoperatorio y de éstos, solo $65 \%$ recibieron quimioterapia postoperatoria. A pesar de ello, las chances de supervivencia libre de enfermedad y supervivencia general fueron mayores para el grupo de quimioterapia. La tasa de supervivencia a 5 años fue 36.3\% (95\% CI, 29.5 a 43.0) para los pacientes tratados con quimioterapia perioperatoria y 23.0 \% (95\% CI, 16.6 a 29.4) para cirugía sola.

El estudio FLOT comparó quimioterapia perioperatoria con epirubicina, cisplatino y 5FU o capecitabina (ECF/ECX) con 5FU, LV, oxaliplatino y docetaxel (FLOT) ${ }^{(14)}$. El grupo experimental con FLOT tuvo una supervivencia media de 50 meses, comparado con 35 meses con ECF/ECX. Ambos grupos tuvieron una tasa similar de efectos adversos serios, hospitalizaciones por toxicidad y mortalidad, que fue menor al $1 \%$.

El estudio CRITICS comparó quimioterapia perioperatoria con quimioterapia preoperatoria seguida de QRT adyuvante $^{(15)}$. Aquí la adición de QRT postoperatoria no mejoró la supervivencia.

\section{Tratamientos neoadyuvantes}

Las terapias neoadyuvantes podrían disminuir la tasa de abandono de tratamiento.

El estudio ACCORD-07 comparó quimioterapia perioperatoria con cisplatino y $5 \mathrm{FU}$ con cirugía sol ${ }^{(16)}$. La quimioterapia preoperatoria aumentó la tasa de resección curativa, y la quimioterapia perioperatoria aumentó la supervivencia general libre de enfermedad. 
El estudio CRITICS II, aún no publicado, intenta determinar la mejor estrategia preoperatoria comparando quimioterapia neoadyuvante vs quimioterapia seguida de QRT preoperatoria $^{(17)}$.

\section{Rol del tratamiento sistémico \\ en el cáncer gástrico avanzado}

Los tratamientos sistémicos se usan para paliar síntomas, aumentar la supervivencia y mejorar la calidad de vida.

Es necesario conocer si hay sobreexpresión del receptor HER 2 (Human Epidermal Growth Factor 2), la expresión de PDL1 (Programmed cell Death Ligand 1), de las enzimas reparadoras del pareo de bases de ADN (Mismatch Repair o MMR) y la inestabilidad de microsatélites (MicroSatelite Instability o MSI), ya que las terapias dirigidas a estos biomarcadores han mejorado la tasa de respuesta y supervivencia.

\section{Terapia anti-HER2}

La amplificación del oncogen HER2, que conlleva a la sobreexpresión del receptor HER2 (ERBB2 o CerbB2), está asociada a proliferación de células tumorales, apoptosis, migración, adhesión y diferenciación. La sobreexpresión de HER2 en el cáncer gástrico o de la unión gastroesofágica varía dependiendo de los diferentes estudios, con una frecuencia del $4.4 \%$ al $53.4 \%$ (media de $17.9 \%)^{(18)}$.

Trastuzumab es un anti-cuerpo monoclonal contra el receptor HER2. El estudio ToGA comparó quimioterapia y trastuzumab con quimioterapia sola en pacientes con cáncer gástrico o de la unión gastroesofágica avanzado HER2 positivo ${ }^{(19)}$. La supervivencia media fue de 16 meses con trastuzumab y quimioterapia comparada con 11.1 meses en pacientes con quimioterapia sola (HR 0.74; 95\% CI 0.60-0.91; $\mathrm{p}=0.0046$ ).

El estudio HERXO analizó la respuesta a la combinación de trastuzumab, capecitabina y oxaliplatino y reportó una respuesta completa en $8.7 \%$, respuesta parcial en $37.8 \%$ y enfermedad estable en $31.1 \%{ }^{(20)}$. Un $4.4 \%$ de pacientes desarrolló reducción asintomática de la fracción de eyección.

Trastuzumab deruxtecan es un nuevo agente contra HER2, un conjugado de anticuerpo monoclonal anti-HER2 unido a un agente citotóxico, que ha mostrado respuestas alentadoras en el cáncer de mama HER2+. El estudio DESTINY-Gastric01 comparó trastuzumab deruxtecan con otras quimioterapias en pacientes con cáncer gástrico avanzado HER2 positivo tratados previamente ${ }^{(21)}$. Se observó respuesta en el $51 \%$ de los pacientes tratados con trastuzumab deruxtecan versus $15 \%$ en el grupo de quimioterapia $(p<0.001)$. La supervivencia general media fue de 12.5 meses con trastuzumab deruxtecan vs 8.4 meses con quimioterapia (HR 0.59; $95 \%$ CI 0.39 a $0.88 ; \mathrm{P}=0.01)$. Los efectos adversos importantes fueron mielosupresión y enfermedad intersticial pulmonar.

\section{Inmunoterapia-inhibidores de puntos de control inmunitarios}

En la superficie de las células tumorales hay sobreexpresión de proteínas PD-L1 y PD-L2. La unión de PD-L1/L2 a la proteína PD1 en los linfocitos $\mathrm{T}$ crea una estimulación negativa e inhibe la respuesta citotóxica, permitiendo al tumor evadir los sistemas de vigilancia inmune. Drogas inhibidoras de puntos de control inmunitarios, como pembrolizumab y nivolumab (anti-PD1), atezolizumab, avelumab y durvalumab (anti-PD-L1) e ipilimumab (CTLA4 - antigeno asociado al linfocito $\mathrm{T}$ citotóxico 4) son capaces de antagonizar la tolerancia inmune y tener un efecto antitumoral ${ }^{(22)}$.

Desde el 2017 está aprobado el uso de pembrolizumab para tumores sólidos metastásicos con deficiencia del sistema de reparo de bases de ADN (dMMR- deficient mismatch repair enzymes) o inestabilidad alta de microsatélites (MSImicrosatellite instability high), que se da como consecuencia de la falla de los mecanismos de reparo del $\mathrm{ADN}^{(23)}$.

El estudio ATTRACTION-2 demostró el beneficio de nivolumab sobre placebo en pacientes asiáticos con cáncer gástrico o de la unión gastroesofágica previamente tratados ${ }^{(24)}$. La supervivencia media fue de 5.26 meses en el grupo con nivolumab y 4.14 meses en el grupo con placebo, que fue una diferencia estadísticamente significativa. La supervivencia general a los 12 meses fue de $27.3 \%$ con nivolumab y $11.6 \%$ con placebo; esto llevó a la aprobación de nivolumab para cáncer gástrico en Japón, Corea del Sur y Taiwán.

Pembrolizumab fue aprobado por la FDA como monoterapia para el tratamiento del cáncer gástrico o de la unión gastroesofagica metastásico tratados previamente, basado en el estudio KEYNOTE-059 ${ }^{(25)}$. La tasa de respuesta a pembrolizumab fue de $11.6 \%$, con respuesta completa en $2.3 \%$. En los pacientes con PD-L1 CPS $>=1$ la tasa de respuesta fue de $22.7 \%$, mientras que en los pacientes con PD-L1 negativo la tasa de respuesta fue de solo $8.6 \%$. Aún más, en los pacientes con MSI-H la tasa de respuesta fue de $57 \%$.

En el estudio KEYNOTE-061, pembrolizumab dado en segunda línea en cáncer gástrico avanzado con PD-L1 positivo no mejoró la tasa de supervivencia comparado con paclitaxel (26). 
El estudio KEYNOTE-062 randomizó a pacientes con cáncer gástrico o de la unión gastroesofágica con $\mathrm{CPS}>=1$ no tratados previamente en 3 grupos: pembrolizumab, pembrolizumab más quimioterapia (cisplatino y fluoropirimidina) o quimioterapia más placebo ${ }^{(27)}$. En pacientes $\operatorname{con} \mathrm{CPS}>=1$, pembrolizumab fue no inferior y no superior a quimioterapia en supervivencia general. Pembrolizumab prolongó la supervivencia general en pacientes con CPS $>10$ pero esto no fue probado estadísticamente. Pembrolizumab más quimioterapia no fue superior a quimioterapia sola en supervivencia general en pacientes con CPS $>=1$. A pesar de que pembrolizumab no mostró beneficio sobre quimioterapia en este grupo de pacientes, fue mejor tolerado que la quimioterapia.

El estudio CheckMate-649, compara nivolumab más ipilimumab vs nivolumab más quimioterapia vs quimioterapia sola, en primera línea ${ }^{(28)}$. Los resultados preliminares fueron presentado en setiembre de $2020^{(29)}$. Nivolumab más quimioterapia en primera línea mejoró la supervivencia general y supervivencia libre de progresión comparado con quimioterapia sola en pacientes con CPS $>5$. La supervivencia media con nivolumab y quimioterapia fue 14.4 vs 11.1 meses con quimioterapia sola. La supervivencia sin progresión de enfermedad fue 7.7 meses con nivolumab y quimioterapia vs 6.1 meses con quimioterapia sola.

El estudio ATTRACTION-4 evalúa nivolumab más quimioterapia versus quimioterapia más placebo como primera línea en cáncer gástrico o de la unión gastroesofágica avanzado en una población asiática ${ }^{(30)}$. Los reportes preliminares mostraron mejores tasas de supervivencia libre de enfermedad y mayor respuesta con la combinación de nivolumab y quimioterapia.

\section{Quimioterapia en primera y segunda línea y tratamiento con anti VEGFR2 (vascular endothelia growth factor receptor 2)}

Se prefiere usar combinaciones de dos agentes citotóxicos en primera línea, ya que es menos tóxico que las combinaciones de 3 drogas. El German Study Group comparó 5FU/oxaliplatino (FLO) con 5FU/Cisplatino (FLP): FLO tuvo menor toxicidad y parece estar asociado a mayor eficacia, supervivencia libre de enfermedad y supervivencia general en pacientes mayores de 65 años $^{(31)}$.

En casos de cáncer con sobreexpresión de HER2, el tratamiento estándar es quimioterapia, (Fluoropirimidina con cisplatino u oxaliplatino) y trastuzumab ${ }^{(19,32)}$.

En casos de tumores que son HER2 negativo, el tratamiento de preferencia en primera línea es quimioterapia con una fluoropirimidina (capecitabina o 5FU) y oxaliplatino o cisplatino $^{(31)}$. Si el PD-L1 CPS es mayor o igual a 5, el nuevo estándar de tratamiento es quimioterapia y nivolumab, basado en los resultados preliminares del estudio Check-Mate $649^{(29) .}$

La combinación de 5FU e irinotecan (FOLFIRI) es una alternativa para la primera línea $^{(33)}$.

Algunas drogas antiangiogénicas también han demostrado beneficio en el tratamiento del cáncer gástrico avanzado. Ramucirumab es un anticuerpo monoclonal humanizado específico para el receptor del factor de crecimiento endotelial (VEGFR2). Ramucirumab fue el primer agente biológico que mostró aumento de la supervivencia en cáncer gástrico avanzado. El estudio REGARD comparó ramucirumab con placebo en segunda línea ${ }^{(34)}$. La supervivencia media fue 5.2 meses para ramucirumab y 3.8 meses para placebo (HR: 0.78; 95\% CI: 0.603-0.998; P = 0.047). El studio RAINBOW comparó ramucirumab con paclitaxel vs paclitaxel sólo en segunda línea ${ }^{(35)}$. La supervivencia media y la supervivencia media libre de enfermedad fueron significativamente más largas con ramucirumab y paclitaxel que con paclitaxel solo ( 9.6 meses vs 7.4 meses y 4.4 vs 2.9 meses, respectivamente). Así, si el paciente puede tolerar quimioterapia se prefiere ramucirumab con quimioterapia en segunda línea.

El estudio TAGS comparó trifluridina/tipiracilo vs placebo en pacientes tratados múltiples veces ${ }^{(36)}$. Trifluridina/tipiracilo demostró ser un tratamiento efectivo en esta población, con reducción del riesgo de muerte de $31 \%$ y mejora de la supervivencia por 2.1 meses.

\section{Conclusión}

La detección temprana y prevención son de suma importancia contra el cáncer. Al entender mejor los mecanismos de enfermedad, cascadas de activación oncogénica y respuesta immune al cáncer seremos capaces de encontrar tratamientos más selectivos, eficaces y tolerables. En la actualidad muchos estudios de investigación están en marcha. Estos estudios ayudarán a identificar biomarcadores predictivos de respuesta a inmunoterapia y otras terapias, y a encontrar tratamientos más efectivos. Se está estudiando inmunoterapia y agentes anti-HER2 en forma adyuvante, quimioterapias más claudiximab (anti-Claudin 18.2), inhibidores del receptor del factor de crecimiento fibroblástico 2, combinaciones de antiangiogénicos o quimioterapias con inhibidores de puntos de control inmunitario, entre otros ${ }^{(37)}$. 
Referencias bibliográficas

1.SEER (Surveillance, Epidemiology and End Results Program). Cancer Statistics Review 1975-2017. NCI.

2. Willy C, Ramirez, et al. Análisis de la situación del cáncer en el Perú, 2018. Centro Nacional de Epidemiologia, Prevención y Control de Enfermedades. Ministerio de Salud del Perú.

3. National Comprehensive Cancer Network (NCCN). Guidelines v1.2021. Gastric cancer.

4. Wanebo HJ, Kennedy BJ, Chmiel J, Steele G Jr, Winchester D, Osteen R. Cancer of the stomach. A patient care study by the American College of Surgeons. Ann Surg. $1993 ; 218(5): 583-592$.

5. Hermans J, Bonenkamp JJ, Boon MC, Bunt AM, Ohyama S, Sasako M, Van de Velde CJ. Adjuvant therapy after curative resection for gastric cancer: meta-analysis of randomized trials. J Clin Oncol. 1993;11(8):1441-1447.

6. Zhang ZX, Gu XZ, Yin WB, Huang GJ, Zhang DW, Zhang RG. Randomized clinical trial on the combination of preoperative irradiation and surgery in the treatment of adenocarcinoma of gastric cardia (AGC)--report on 370 patients. Int J Radiat Oncol Biol Phys. 1998;42(5):929-934.

7. Macdonald JS, Smalley SR, Benedetti J, Hundahl SA, Estes NC, Stemmermann GN, et al. Chemoradiotherapy after surgery compared with surgery alone for adenocarcinoma of the stomach or gastroesophageal junction. N Engl J Med. 2001 ;345(10):725-730.

8. Fuchs CS, Niedzwiecki D, Mamon HJ, Tepper JE, Ye X, Swanson RS, et al. Adjuvant Chemoradiotherapy With Epirubicin, Cisplatin, and Fluorouracil Compared With Adjuvant Chemoradiotherapy With Fluorouracil and Leucovorin After Curative Resection of Gastric Cancer: Results From CALGB 80101 (Alliance). J Clin Oncol. 2017;35(32):3671-3677.

9. Park SH, Sohn TS, Lee J, Lim DH, Hong ME, Kim KM, et al. Phase III Trial to Compare Adjuvant Chemotherapy With Capeci- tabine and Cisplatin Versus Concurrent Chemoradiotherapy in Gastric Cancer: Final Report of the Adjuvant Chemoradiotherapy in Stomach Tumors Trial, Including Survival and Subset Analyses. J Clin Oncol. 2015;33(28):3130-3136.

10. Park SH, Lim DH, Sohn TS, Lee J, Zang DY, Kim ST, et al. ARTIST 2 investigators. A randomized phase III trial comparing adjuvant single-agent $\mathrm{S} 1, \mathrm{~S}-1$ with oxaliplatin, and postoperative chemoradiation with $\mathrm{S}-1$ and oxaliplatin in patients with node-positive gastric cancer after D2 resection: the ARTIST 2 trial?. Ann Oncol. 2021;32(3):368-374.

11. Sakuramoto S, Sasako M, Yamaguchi T, Kinoshita T, Fujii M, Nashimoto A, et al. ACTS-GC Group. Adjuvant chemotherapy for gastric cancer with S-1, an oral fluoropyrimidine. N Engl J Med. 2007;357(18):1810-1820.

12. Bang YJ, Kim YW, Yang HK, Chung HC, Park YK, Lee $\mathbf{K H}$, et al. CLASSIC trial investigators. Adjuvant capecitabine and oxaliplatin for gastric cancer after D2 gastrectomy (CLASSIC): a phase 3 open-label, randomized controlled trial. Lancet. 2012;379(9813):315-321.

13. Cunningham D, Allum WH, Stenning SP, Thompson JN, Van de Velde CJ, Nicolson $M$, et al. MAGIC Trial Participants. Perioperative chemotherapy versus surgery alone for resectable gastroesophageal cancer. $\mathrm{N}$ Engl J Med. 2006;355(1):11-20.

14. Al-Batran SE, Homann N, Pauligk C, Goetze TO, Meiler J,
Kasper S, et al. FLOT4-AIO Investigators. Perioperative chemotherapy with fluorouracil plus leucovorin, oxaliplatin, and docetaxel versus fluorouracil or capecitabine plus cisplatin and epirubicin for locally advanced, resectable gastric or gastro-oesophageal junction adenocarcinoma (FLOT4): a randomised, phase $2 / 3$ trial. Lancet 15 .

15. Cats A, Jansen EPM, van Grieken NCT, Sikorska K, Lind P, Nordsmark M, et al. CRITICS investigators. Chemotherapy versus chemoradiotherapy after surgery and preoperative chemotherapy for resectable gastric cancer (CRITICS): an international, open-label, randomised phase 3 trial. Lancet Oncol. 2018;19(5):616-628.

16.Ychou M, Boige V, Pignon JP, Conroy T, Bouché O, Lebreton G, et al. Perioperative chemotherapy compared with surgery alone for resectable gastroesophageal adenocarcinoma: an FNCLCC and FFCD multicenter phase III trial. J Clin Oncol. 2011;29(13):1715-1721.

17.Slagter AE, Jansen EPM, van Laarhoven HWM, van Sandick JW, van Grieken NCT, Sikorska K, et al. CRITICSII: a multicentre randomised phase II trial of neo-adjuvant chemotherapy followed by surgery versus neo-adjuvant chemotherapy and subsequent chemoradiotherapy followed by surgery versus neo-adjuvant chemoradiotherapy followed by surgery in resectable gastric cancer. BMC Cancer. 2018;18(1):877.

18.Abrahao-Machado LF, Scapulatempo-Neto C. HER2 testing in gastric cancer: An update. World J Gastroenterol. 2016;22(19):4619-4625.

19.Bang YJ, Van Cutsem E, Feyereislova A, Chung HC, Shen L, Sawaki A, et al. ToGA Trial Investigators. Trastuzumab in combination with chemotherapy versus chemotherapy alone for treatment of HER2-positive advanced gastric or gastrooesophageal junction cancer (ToGA): a phase 3, open-label, randomized controlled trial. Lancet. 2010;376(9742):687-697.

20.Rivera F, Romero C, Jimenez-Fonseca P, IzquierdoManuel M, Salud A, Martínez E, et al. Phase II study to evaluate the efficacy of Trastuzumab in combination with Capecitabine and Oxaliplatin in first-line treatment of HER2positive advanced gastric cancer: HERXO trial. Cancer Chemother Pharmacol. 2019;83(6):1175-1181.

21.Shitara K, Bang YJ, Iwasa S, Sugimoto N, Ryu MH, Sakai D, et al. DESTINY-Gastric01 Investigators. Trastuzumab Deruxtecan in Previously Treated HER2-Positive Gastric Cancer. N Engl J Med. 2020;382(25):2419-2430.

22.Bonelli P, Borrelli A, Tuccillo FM, Silvestro L, Palaia R, Buonaguro FM. Precision medicine in gastric cancer. World J Gastrointest Oncol. 20195;11(10):804-829.

23.https://www.fda.gov/drugs/resources-informationapproved-drugs/fda-grants-accelerated-approvalpembrolizumab-first-tissuesite-agnostic-indication

24.Kang YK, Boku N, Satoh T, Ryu MH, Chao Y, Kato K, et al. Nivolumab in patients with advanced gastric or gastrooesophageal junction cancer refractory to, or intolerant of, at least two previous chemotherapy regimens (ONO-4538-12, ATTRACTION-2): a randomised, double-blind, placebocontrolled, phase 3 trial. Lancet. 2017;390(10111):2461-2471.

25. Fuchs CS, Doi T, Jang RW, Muro K, Satoh T, Machado M, et al. Safety and Efficacy of Pembrolizumab Monotherapy in Patients With Previously Treated Advanced Gastric and Gastroesophageal Junction Cancer: Phase 2 Clinical 
KEYNOTE-059 Trial. JAMA Oncol. 2018;4(5):e180013.

26. Shitara K, Özgüroğlu M, Bang YJ, Di Bartolome M, Mandalà M, Ryu MH, et al. KEYNOTE-061 investigators. Pembrolizumab versus paclitaxel for previously treated, advanced gastric or gastro-oesophageal junction cancer (KEYNOTE-061): a randomised, open-label, controlled, phase 3 trial. Lancet. 2018;392(10142):123-133.

27. Shitara K, Van Cutsem E, Bang YJ, Fuchs C, Wyrwicz L, Lee KW, et al. Efficacy and Safety of Pembrolizumab or Pembrolizumab Plus Chemotherapy vs Chemotherapy Alone for Patients With First-line, Advanced Gastric Cancer: The KEYNOTE-062 Phase 3 Randomized Clinical Trial. JAMA Oncol. 2020;6(10):1571-1580.

28. ClinicalTrials.gov identifier (NCT number): NCT02872116

29. Moehler M, Shitara K, Garrido M, Salman $P$, Shen $L$, Wyrwicz L, et al. LBA6_PR - Nivolumab (nivo) plus chemotherapy (chemo) versus chemo as first-line (1L) treatment for advanced gastric cancer/gastroesophageal junction cancer (GC/GEJC)/esophageal adenocarcinoma (EAC): First results of the CheckMate 649 study. ESMO Sep 2020 - Presidential Symposium. Ann Oncol 2020;31:S1191.

30. Boku N, Ryu MH, Oh D, Oh SC, Chung HC, Lee K, et al. LBA7 PR - Nivolumab plus chemotherapy versus chemotherapy alone in patients with previously untreated advanced or recurrent gastric/gastroesophageal junction (G/GEJ) cancer: ATTRACTION-4 (ONO-4538-37) study. ESMO Sep 2020 - Presidential Symposium.

31.Al-Batran SE, Hartmann JT, Probst S, Schmalenberg H, Hollerbach S, Hofheinz R, et al. Arbeitsgemeinschaft Internistische Onkologie. Phase III trial in metastatic gastroesophageal adenocarcinoma with fluorouracil, leucovorin plus either oxaliplatin or cisplatin: a study of the Arbeitsgemeinschaft Internistische Onkologie. J Clin Oncol. 2008;26(9):1435-1442.
32. Ryu MH, Yoo C, Kim JG, Ryoo BY, Park YS, Park SR, et al. Multicenter phase II study of trastuzumab in combination with capecitabine and oxaliplatin for advanced gastric cancer. Eur J Cancer. 2015;51(4):482-488.

33. Guimbaud R, Louvet C, Ries P, Ychou M, Maillard E, André T, et al. Prospective, randomized, multicenter, phase III study of fluorouracil, leucovorin, and irinotecan versus epirubicin, cisplatin, and capecitabine in advanced gastric adenocarcinoma: a French intergroup (Fédération Francophone de Cancérologie Digestive, Fédération Nationale des Centres de Lutte Contre le Cancer, and Groupe Coopérateur Multidisciplinaire en Oncologie) study. J Clin Oncol. 2014; 32(31):3520-3526.

34. Fuchs CS, Tomásek J, Yong CJ, Dumitru F, Passalacqua R, Goswami C, et al. REGARD Trial Investigators. Ramucirumab monotherapy for previously treated advanced gastric or gastro-oesophageal junction adenocarcinoma (REGARD): an international, randomised, multicentre, placebo-controlled, phase 3 trial. Lancet. 2014;383(9911):3139.

35. Wilke H, Muro K, Van Cutsem E, Oh SC, Bodoky G, Shimada Y, et al. RAINBOW Study Group. Ramucirumab plus paclitaxel versus placebo plus paclitaxel in patients with previously treated advanced gastric or gastro-oesophageal junction adenocarcinoma (RAINBOW): a double-blind, randomised phase 3 trial. Lancet Oncol. 2014;15(11):12241235.

36.Shitara K, Doi T, Dvorkin M, Mansoor W, Arkenau HT, Prokharau A, et al. Trifluridine/tipiracil versus placebo in patients with heavily pretreated metastatic gastric cancer (TAGS): a randomized, double-blind, placebo-controlled, phase 3 trial. Lancet Oncol. 2018;19(11):1437-1448.

37.Smyth EC, Nilsson M, Grabsch HI, van Grieken NC, Lordick F. Gastric cáncer. Lancet. 2020;396(10251):635-648.

Contribución de autoría: Natalia Ferrari ha sido la autora de esta publicación, contribuyendo a su concepción, organización de la presentación científica, búsqueda electrónica, revisión inicial, redacción y revisión final.

Conflicto de interés: El autor no tiene conflicto de interés con la publicación de este trabajo.

Financiamiento: Autofinanciado.

Citar como: Ferrari, N. Rol del tratamiento sistémico en el cáncer gástrico. Diagnóstico(Lima). 2021;60(2):92-97.

DOI: 10.33734 /diagnostico.v60i2.286

Correspondencia: Natalia Ferrari. Correo electrónico: nataliaferrari@hotmail.com

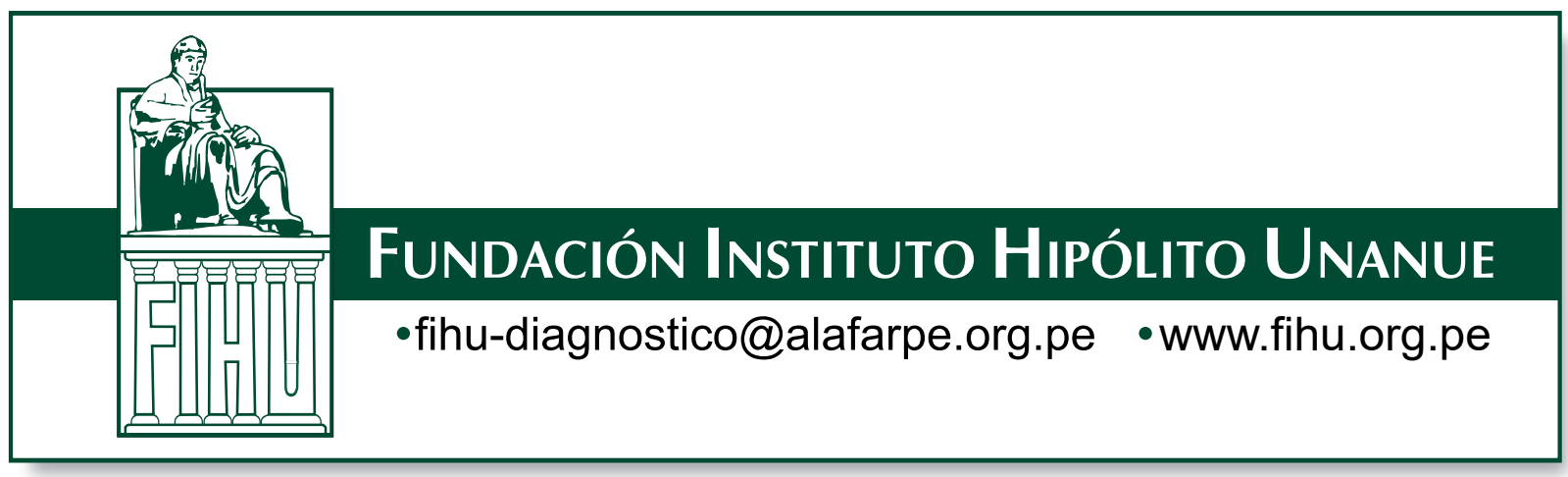

